\title{
Bethe graphs attached to the vertices of a connected graph - a spectral approach
}

\author{
Enide Andrade ${ }^{1}$, Domingos M. Cardoso*1, Luis Medina $^{2}$, and \\ Oscar Rojo ${ }^{3}$ \\ ${ }^{1}$ CIDMA - Centro de Investigação e Desenvolvimento em Matemática e Aplicações, \\ Departamento de Matemática, Universidade de Aveiro, Aveiro, Portugal. Email: \\ (enide,dcardoso)@ua.pt \\ ${ }^{2}$ Departamento de Matemáticas, Universidad de Antofagasta, Antofagasta, Chile. \\ Email: luis.medina@uantof.cl \\ ${ }^{3}$ Departamento de Matemáticas, Universidad Católica del Norte, Antofagasta, Chile. \\ Email: orojo@ucn.cl
}

May 2, 2016

\begin{abstract}
A weighted Bethe graph $B$ is obtained from a weighted generalized Bethe tree by identifying each set of children with the vertices of a graph belonging to a family $F$ of graphs. The operation of identifying the root vertex of each of $r$ weighted Bethe graphs to the vertices of a connected graph $\mathcal{R}$ of order $r$ is introduced as the $\mathcal{R}$-concatenation of a family of $r$ weighted Bethe graphs. It is shown that the Laplacian eigenvalues (when $F$ has arbitrary graphs) as well as the signless Laplacian and adjacency eigenvalues (when the graphs in $F$ are all regular) of the $\mathcal{R}$-concatenation of a family of weighted Bethe graphs can be computed (in a unified way) using the stable and low computational cost methods available for the determination of the eigenvalues of symmetric tridiagonal matrices. Unlike the previous results already obtained on this topic, the more general context of families of distinct weighted Bethe graphs is herein considered.
\end{abstract}

AMS Subject Classification: 05C50, 05C76, 15A18.

Keywords: Graph spectra, graph operations, Laplacian matrix, signless Laplacian matrix, adjacency matrix.

\footnotetext{
*Corresponding author.
} 


\section{Introduction}

\subsection{Basic notation and definitions}

In this paper we deal with undirected simple graphs $\mathcal{G}$ of order $n$, with vertex set $V(\mathcal{G})=\{1, \ldots, n\}$ and edge set $E(\mathcal{G})$. Considering a vertex subset $S \subset V(\mathcal{G})$, $\partial(S)$ denotes the subset of edges that have exactly one vertex in $S$. Throughout it is assumed that each edge $e \in E(\mathcal{G})$ has a positive weight $w(e)$. When $w(e)=1$ for all $e \in E(\mathcal{G})$ the graph $\mathcal{G}$ is an unweighted graph otherwise is weighted. The adjacency matrix of a graph $\mathcal{G}$ is the $n \times n$ matrix $A(\mathcal{G})=\left(a_{i, j}\right)$ such that $a_{i, j}=w(i j)$ if there is an edge $i j$ joining the vertices $i$ and $j$ and $a_{i, j}=0$ elsewhere. The Laplacian matrix and the signless Laplacian matrix of $\mathcal{G}$ are $L(\mathcal{G})=D(\mathcal{G})-A(\mathcal{G})$ and $Q(\mathcal{G})=D(\mathcal{G})+A(\mathcal{G})$, respectively, where $D(\mathcal{G})$ is the diagonal matrix with diagonal entries $d_{i, i}=\sum_{e \in \partial(\{i\})} w(e)$, where the sum is taken over all edges incident to the vertex $i$. Notice that the matrices $L(\mathcal{G}), Q(\mathcal{G})$ and $A(\mathcal{G})$ are real and symmetric and thus all of their eigenvalues are real. Furthermore, $L(\mathcal{G})$ and $Q(\mathcal{G})$ are positive semidefinite and hence each of their eigenvalues is nonnegative.

Let $\mathcal{B}$ be a $\hat{w}$-weighted generalized Bethe tree with $k>1$ levels, which is a rooted tree in which the vertices at the same level have the same degree and edges connecting vertices at consecutive levels $(k-j+1$ and $k-j)$ have the same weight $w_{j}$ (the $j$-th entry of the $(k-1)$-tuple $\hat{w}$ ), with $1 \leq j \leq k-1$. When all of the weights are equal to $1, \mathcal{B}$ is called an unweighted generalized Bethe tree or simply a generalized Bethe tree. Considering a family of graphs $F=\left\{\mathcal{G}_{j}: j \in \Delta\right\}$, where $\Delta$ is a non-empty index subset of $\{1, \ldots, k-1\}$, the graph $\mathcal{B}(F)$ obtained from the $\hat{w}$-weighted generalized Bethe tree $\mathcal{B}$ by identifying each set of children of $\mathcal{B}$ at level $k-j+1$ with the vertices of the graph $\mathcal{G}_{j} \in F$, is herein called a $\hat{w}$-weighted Bethe graph. This graph is called an unweighted Bethe graph or simply a Bethe graph when all the weights in the $(k-1)$-tuple $\hat{w}$ are equal to 1 (see [2, Fig.1.2] as an example). The root vertex of $\mathcal{B}(F)$ coincides with the root vertex of $\mathcal{B}$. For $1 \leq j \leq k, n_{j}$ and $d_{j}$ are the number and the degree of the vertices of $\mathcal{B}$ at the level $k-j+1$, respectively. We recall that for a rooted graph the level of a vertex is one more than its distance from the root vertex. Thus $d_{k}$ is the degree of the root vertex, $n_{k}=1, d_{1}=1$ and $n_{1}$ is the number of pendant vertices. Let us consider

$$
\delta_{j}= \begin{cases}w_{j} & \text { if } j=1 \\ \left(d_{j}-1\right) w_{j-1}+w_{j} & \text { if } 2 \leq j \leq k-1, \\ d_{k} w_{k-1} & \text { if } j=k\end{cases}
$$

Notice that $\delta_{j}$ is the sum of the weights of the edges of $\mathcal{B}$ incident with a vertex at the level $k-j+1$. If $w_{1}=w_{2}=\cdots=w_{k-1}=1$, then $\delta_{j}=d_{j}$ for $j=1, \ldots, k$. 
Now, let $m_{j}=\frac{n_{j}}{n_{j+1}}$ for $j=1, \ldots, k-1$. Then

$$
\begin{aligned}
m_{j} & =d_{j+1}-1(1 \leq j \leq k-2) \\
d_{k} & =n_{k-1}=m_{k-1}
\end{aligned}
$$

where $m_{j}$ is the cardinality of each set of children at the level $k-j+1$.

From now on, $M=M(\mathcal{G})$ is one of the matrices $L(\mathcal{G}), Q(\mathcal{G})$ or $A(\mathcal{G})$. We consider only matrices $M_{j}=M\left(\mathcal{G}_{j}\right)$, with eigenvalues $\theta_{1}\left(M_{j}\right), \ldots, \theta_{m_{j}}\left(M_{j}\right)$, for which $\mathbf{e}_{m_{j}}$ (the all one vector with $m_{j}$ entries) is an eigenvector associated to the eigenvalue $\theta_{m_{j}}\left(M_{j}\right)$, that is,

$$
M_{j} \mathbf{e}_{m_{j}}=\theta_{m_{j}}\left(M_{j}\right) \mathbf{e}_{m_{j}} .
$$

Observe that (1) holds if $M\left(\mathcal{G}_{j}\right)=L\left(\mathcal{G}_{j}\right)$ and also if $M\left(\mathcal{G}_{j}\right)=Q\left(\mathcal{G}_{j}\right)$ or $M\left(\mathcal{G}_{j}\right)=$ $A\left(\mathcal{G}_{j}\right)$ whenever $\mathcal{G}_{j}$ is a regular graph. We introduce the notation

$$
s=\left\{\begin{array}{cl}
-1 & \text { if } M \text { is the Laplacian matrix } \\
1 & \text { if } M \text { is the signless Laplacian or the adjacency matrix }
\end{array}\right.
$$

and

$$
a= \begin{cases}0 & \text { if } M \text { is the adjacency matrix } \\ 1 & \text { if } M \text { is the Laplacian or the signless Laplacian matrix. }\end{cases}
$$

Definition 1.1 Let us define the matrices $X_{j}$, for $j=1, \ldots, k-1$, and the matrices $X_{j, l}$ for $j=1,2, \ldots, k-1$ and $l=1, \ldots, m_{j}-1$, as follows. $X_{j}$ is the $j \times j$ leading principal submatrix of the $k \times k$ symmetric tridiagonal matrix $X_{k}=$

$$
\left[\begin{array}{ccccc}
a \delta_{1}+\theta_{m_{1}}\left(M_{1}\right) & w_{1} \sqrt{m_{1}} & & & \\
w_{1} \sqrt{m_{1}} & a \delta_{2}+\theta_{m_{2}}\left(M_{2}\right) & \ddots & & \\
& \ddots & \ddots & w_{k-2} \sqrt{m_{k-2}} & \\
& & w_{k-2} \sqrt{m_{k-2}} & a \delta_{k-1}+\theta_{m_{k-1}}\left(M_{k-1}\right) & w_{k-1} \sqrt{m_{k-1}} \\
& & & w_{k-1} \sqrt{m_{k-1}} & a \delta_{k}
\end{array}\right]
$$

and

$$
X_{j, l}=\left[\begin{array}{ccccc}
a \delta_{1}+\theta_{m_{1}}\left(M_{1}\right) & w_{1} \sqrt{m_{1}} & & & \\
w_{1} \sqrt{m_{1}} & \ddots & \ddots & & \\
& \ddots & \ddots & w_{j-2} \sqrt{m_{j-2}} & \\
& & w_{j-2} \sqrt{m_{j-2}} & a \delta_{j-1}+\theta_{m_{j-1}}\left(M_{j-1}\right) & w_{j-1} \sqrt{m_{j-1}} \\
& & & w_{j-1} \sqrt{m_{j-1}} & a \delta_{j}+\theta_{l}\left(M_{j}\right)
\end{array}\right]
$$

The multiset of eigenvalues of a square matrix $C$ is denoted $\sigma(C)$. 


\subsection{Previous results and the aim of the paper}

Several results on the spectra of matrices associated to generalized Bethe trees were published in $[3,7,11]$ and some of those results were generalized in [12]. In fact, assuming (1) and defining $\Omega=\left\{j: 1 \leq j \leq k-1, n_{j}>n_{j+1}\right\}$, we have the following result stated in [12, Th. 2.10].

Theorem 1.2 [12]

$\sigma(M(\mathcal{B}(\mathrm{F})))=\sigma\left(X_{k}\right) \cup\left(\cup_{j \in \Omega-\Delta} \sigma\left(X_{j}\right)^{n_{j}-n_{j+1}}\right) \cup\left(\cup_{j \in \Delta}\left(\cup_{l=1}^{m_{j}-1} \sigma\left(X_{j, l}\right)^{n_{j+1}}\right)\right)$,

where $\sigma\left(X_{j}\right)^{n_{j}-n_{j+1}}$ and $\sigma\left(X_{j, l}\right)^{n_{j+1}}$ mean that each eigenvalue in $\sigma\left(X_{j}\right)$ and in $\sigma\left(X_{j, l}\right)$ must be considered with multiplicity $n_{j}-n_{j+1}$ and $n_{j+1}$, respectively. Furthermore, the multiplicities of equal eigenvalues obtained in different matrices (if any), must be added.

Denoting by $\widetilde{A}$ the submatrix obtained from a square matrix $A$ by deleting its last row and its last column, from the proofs of Lemma 2.2, Theorem 2.5 and Lemma 2.7 in [12], we obtain

Lemma $1.3[12]$

$$
\mid \lambda I-\widetilde{M(\mathcal{B}(\mathrm{F})}) \mid=D_{k-1}(\lambda) \prod_{j \in \Omega-\Delta}\left(D_{j}(\lambda)\right)^{n_{j}-n_{j+1}} \prod_{j \in \Delta} \prod_{l=1}^{m_{j}-1}\left(D_{j, l}(\lambda)\right)^{n_{j+1}}
$$

where, for $j=1, \ldots, k-1$ and $l=1, \ldots, m_{j}-1, D_{j}(\lambda)$ and $D_{j, l}(\lambda)$ are the characteristic polynomials of the matrices $X_{j}$ and $X_{j, l}$, respectively.

Theorem 1.2 above was extended in $[2$, Th. 3.7] to a graph $\mathcal{R}\{\mathcal{B}(F)\}$ obtained from a connected weighted graph $\mathcal{R}$ on $r$ vertices and $r$ copies of $\mathcal{B}(F)$ by identifying the root of the $i$-th copy $\mathcal{B}(F)$ with the $i$-th vertex of $\mathcal{R}$. Those spectra were given by the eigenvalues of symmetric tridiagonal matrices of order $j$, with $1 \leq j \leq k$, where $k$ is the number of levels of the generalized Bethe tree $\mathcal{B}$. Thus, the spectra of $M(\mathcal{B}(F))$ and $M(\mathcal{R}\{\mathcal{B}(F)\})$, respectively, can be computed using the stable and low computational cost methods available for the determination of the eigenvalues of symmetric tridiagonal matrices (see [6]).

The purpose of this paper is to extend the results obtained in [2] to a graph $\mathcal{R}\left\{\mathcal{H}_{i}: 1 \leq i \leq r\right\}$ defined as follows.

Definition 1.4 Let $\mathcal{R}$ be a connected graph on $r$ vertices $v_{1}, \ldots, v_{r}$. For each $i \in\{1, \ldots, r\}$ consider an index subset $\Delta_{i} \subseteq\left\{1, \ldots, k_{i}-1\right\}$, a family of graphs $\mathrm{F}_{i}=\left\{\mathcal{G}_{i, j}: j \in \Delta_{i}\right\}$ and the $\hat{w}_{i}$-weighted Bethe graph $\mathcal{H}_{i}=\mathcal{B}_{i}\left(\mathrm{~F}_{i}\right)$, where $\mathcal{B}_{i}$ is a $\hat{w}_{i}$-weighted generalized Bethe tree with $k_{i}$ levels. The weighted $\mathcal{R}$-concatenation of the family of weighted Bethe graphs $\mathcal{H}=\left\{\mathcal{H}_{i}: 1 \leq i \leq r\right\}$ is the graph $\mathcal{R}\{\mathcal{H}\}$ obtained from $\mathcal{R}$ and the graphs $\mathcal{H}_{1}, \mathcal{H}_{2}, \ldots, \mathcal{H}_{r}$ by identifying the root of each $\mathcal{H}_{i}$ with $v_{i}$. 
As usually, when the weights in the tuples $\hat{w}_{1}, \ldots, \hat{w}_{r}$ are all equal to 1 the graph $\mathcal{R}\{\mathcal{H}\}$ is called an unweighted $\mathcal{R}$-concatenation or simply a $\mathcal{R}$-concatenation of the family of Bethe graphs $\mathcal{H}$.

The graph depicted in Figure 1 is the $\mathcal{R}$-concatenation of a family of Bethe graphs $\mathcal{H}_{1}, \ldots, \mathcal{H}_{4}$, where $\mathcal{R}$ is obtained from $K_{4}$ after deleting one edge and $\mathcal{H}_{1}, \ldots, \mathcal{H}_{4}$ denotes a collection of four distinct Bethe graphs. Notice that, unlike the case studied in [2], the graphs $\mathcal{H}_{i}$ in $\mathcal{R}\left\{\mathcal{H}_{i}: 1 \leq i \leq r\right\}$ can be distinct.

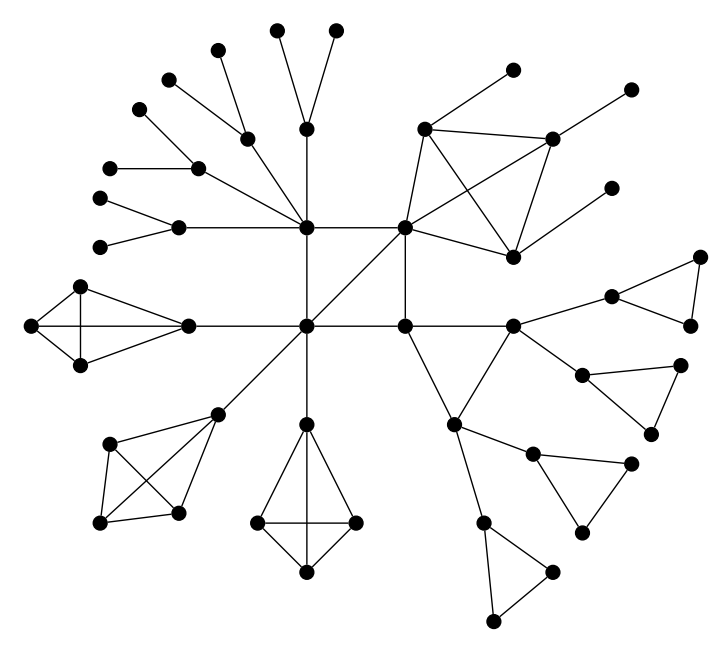

Figure 1: The $\mathcal{R}$-concatenation of a family $\mathcal{H}$ of four Bethe graphs, where $\mathcal{R}$ is obtained from $K_{4}$ after deleting one edge.

A similar operation with the designation of concatenation between two graphs by their root vertices is used in computer science in context-free word grammars [4] and graph codes [1]. This graph operation is also called in [8] the coalescence of two graphs with respect to some particular vertices (one vertex in each graph).

The remaining sections of this paper are organized as follows. Section 2 is devoted to the introduction of some additional notation to facilitate the presentation of the main result of Section 3. In Section 3 we characterize the eigenvalues, including their multiplicities, of the matrix $M(\mathcal{R}\{\mathcal{H}\})$ in a unified way. As an application of this main result, in Section 4 the eigenvalues of the Laplacian matrix of the graph $\mathcal{R}\{\mathcal{H}\}$ are characterized as well as the eigenvalues of the signless Laplacian and adjacency matrices of this graph, whenever for $i=1, \ldots, r$, in each family $F_{i}$ (considered for $\mathcal{H}_{i}=\mathcal{B}_{i}\left(F_{i}\right)$ ) the graphs $\mathcal{G}_{i, j}$, with $j \in \Delta_{i}$, are all regular. 


\section{Additional notation}

Before going further it is worth to introduce the following additional notation.

1. For each $i \in\{1, \ldots, r\} \mathcal{H}_{i}=\mathcal{B}_{i}\left(F_{i}\right)$, where $\mathcal{B}_{i}$ is a weighted generalized Bethe tree with $k_{i}$ levels.

(a) For $j=1, \ldots, k_{i}, n_{i, j}$ and $d_{i, j}$ denote the number and the degree of the vertices of $\mathcal{B}_{i}$ at the level $k_{i}-j+1$, respectively.

(b) For $j=1, \ldots, k_{i}-1$, the edges of $\mathcal{B}_{i}$ connecting vertices at the level $k_{i}-j+1$ with the vertices at the level $k_{i}-j$ have the weight $w_{i, j}$.

(c) For $j \in \Delta_{i}$, the edges of the graph $\mathcal{G}_{i, j}$ (defined on the set of children of $\mathcal{B}_{i}$ at level $k_{i}-j+1$ ) have the weight $u_{i, j}$. On the other hand, when $j \notin \Delta_{i}, u_{i, j}=0$.

2. Now let us define $\mathcal{G}=\mathcal{R}\{\mathcal{H}\}$, with $\mathcal{H}=\left\{\mathcal{H}_{i}: 1 \leq i \leq r\right\}$.

(a) Considering $v_{1}, \ldots, v_{r} \in V(\mathcal{R})$, as usually, $v_{i} \sim v_{j}$ means that $v_{i}$ and $v_{j}$ are adjacent.

(b) Furthermore, for every pair of vertices $v_{i}$ and $v_{j}$ if $v_{i} \sim v_{j}$ then $\varepsilon_{i, j}=$ $\varepsilon_{j, i}$ is the weight of the edge $v_{i} v_{j}$, otherwise $\varepsilon_{i, j}=\varepsilon_{j, i}=0$.

(c) For $i=1, \ldots, r$, the degree of $v_{i}$ as a vertex of $\mathcal{R}$ is $d\left(v_{i}\right)$ and $n_{i}=$ $\sum_{j=1}^{k_{i}} n_{i, j}$ is the number of vertices of the graph $\mathcal{H}_{i}$. Therefore, the total number of vertices of $\mathcal{G}$ is $n=\sum_{i=1}^{r} n_{i}$.

(d) For $1 \leq i \leq r$ and $1 \leq j \leq k_{i}-1, m_{i, j}=\frac{n_{i, j}}{n_{i, j+1}}$. Observe that $m_{i, j}$ is the cardinality of each set of children of $\mathcal{B}_{i}$ at the level $k_{i}-j+1$ and then the order of $\mathcal{G}_{i, j}$ is $m_{i, j}$. Moreover

$$
\begin{aligned}
m_{i, j} & =d_{i, j+1}-1, \quad 1 \leq j \leq k_{i}-2 \\
m_{i, k_{i}-1} & =d_{i, k_{i}}=n_{i, k_{i}-1} .
\end{aligned}
$$

(e) For $i=1,2, \ldots, r$, let

$$
\begin{aligned}
\delta_{i, 1} & =w_{i, 1} \\
\delta_{i, j} & =\left(d_{i, j}-1\right) w_{i, j-1}+w_{i, j}, j=2, \ldots, k_{i}-1 \\
\delta_{i, k_{i}} & =d_{i, k_{i}} w_{i, k_{i}-1} \\
\varepsilon_{i} & =\sum_{v_{i} \sim v_{j}} \varepsilon_{i, j} .
\end{aligned}
$$

Observe that if $\mathcal{B}_{i}$ is an unweighted tree then $\delta_{i, j}=d_{i, j}$ and if $\mathcal{R}$ is an unweighted graph then $\varepsilon_{i}=d\left(v_{i}\right)$. 
The identity matrix of appropriate order is denoted by $I$ and $I_{m}$ denotes the identity matrix of order $m$. For further notation the reader is referred to [13] (regarding matrix theory) and [5] (regarding graph spectra).

It should be noted that $\mathcal{B}_{i}$ and $\mathcal{H}_{i}$ have the same vertex set, furthermore the vertices of $\mathcal{G}$ are labeled as follows:

(1) The vertices of $\mathcal{H}_{1}$ are labeled from the bottom to the vertex $v_{1}$, using the labels $1, \ldots, n_{1}$ and at each level from the left to the right;

(2) The vertices of $\mathcal{H}_{2}$ are labeled from the bottom to the vertex $v_{2}$, using the labels $n_{1}+1, \ldots, n_{1}+n_{2}$ and at each level from the left to the right;

(r) The vertices of $\mathcal{H}_{r}$ are labeled from the bottom to the vertex $v_{r}$, using the labels $n-n_{r}+1, \ldots, n$ and at each level from the left to the right.

In addition, for $i=1, \ldots, r$ and $j=1, \ldots, r$, we denote by $E_{i, j}=\left(e_{p q}\right)$ the matrix of order $t_{i} \times t_{j}$ such that $e_{t_{i} t_{j}}=1$ and the other entries are zero.

Using the above mentioned labeling the Laplacian, signless Laplacian and adjacency matrices of $\mathcal{G}$ become $M(\mathcal{G})=$

$$
\left[\begin{array}{ccccc}
M\left(\mathcal{H}_{1}\right)+a \varepsilon_{1} E_{1,1} & s \varepsilon_{1,2} E_{1,2} & \cdots & \cdots & s \varepsilon_{1, r} E_{1, r} \\
s \varepsilon_{1,2} E_{1,2}^{T} & \ddots & \ddots & & s \varepsilon_{2, r} E_{2, r} \\
\vdots & \ddots & \ddots & \ddots & \vdots \\
\vdots & & \ddots & M\left(\mathcal{H}_{r-1}\right)+a \varepsilon_{r-1} E_{r-1, r-1} & s \varepsilon_{r-1, r} E_{r-1, r} \\
s \varepsilon_{1, r} E_{1, r}^{T} & s \varepsilon_{2, r} E_{2, r}^{T} & \cdots & s \varepsilon_{r-1, r} E_{r-1, r}^{T} & M\left(\mathcal{H}_{r}\right)+a \varepsilon_{r} E_{r, r}
\end{array}\right],
$$

where $s$ and $a$ are as in (2) and (3), respectively. On the other hand, for $i=1, \ldots, r, M\left(\mathcal{H}_{i}\right)+a \varepsilon_{i} E_{i, i}=$

$$
\left[\begin{array}{ccccc}
I_{n_{i, 2}} \otimes S_{i, 1} & s I_{n_{i, 2}} \otimes w_{i, 1} \mathbf{e}_{m_{i, 1}} & & & \\
s I_{n_{i, 2}} \otimes w_{i, 1} \mathbf{e}_{m_{i, 1}}^{T} & \ddots & \ddots & & \\
& \ddots & \ddots & \ddots & \\
& & \ddots & S_{i, k_{i}-1} & s w_{i, k_{i}-1} \mathbf{e}_{m_{i, k_{i}-1}} \\
& & & s w_{i, k_{i}-1} \mathbf{e}_{m_{i, k_{i}-1}}^{T} & a\left(\delta_{i, k_{i}}+\varepsilon_{i}\right)
\end{array}\right]
$$

where, for $j=1, \ldots, k_{i}-1$,

$$
S_{i, j}=a \delta_{i, j} I_{m_{i, j}}+M\left(\mathcal{G}_{i, j}\right)
$$

with $M\left(\mathcal{G}_{i, j}\right)=0$ if $j \notin \Delta_{i}$.

Let us assume that $M_{i, j}=M\left(G_{i, j}\right)$ has the eigenvalues $\theta_{1}\left(M_{i, j}\right), \ldots, \theta_{m_{i, j}}\left(M_{i, j}\right)$ such that

$$
M_{i, j} \mathbf{e}_{m_{i, j}}=\theta_{m_{i, j}}\left(M_{i, j}\right) \mathbf{e}_{m_{i, j}} .
$$

This is always the case if $M\left(\mathcal{G}_{i, j}\right)=L\left(\mathcal{G}_{i, j}\right)$ and also the case if $M\left(\mathcal{G}_{i, j}\right)=Q\left(\mathcal{G}_{i, j}\right)$ or $M\left(\mathcal{G}_{i, j}\right)=A\left(\mathcal{G}_{i, j}\right)$ whenever $\mathcal{G}_{i, j}$ is regular. 
Definition 2.1 For $i=1, \ldots, r$ and $j=1, \ldots, k_{i}-1$, let $X_{i, j}$ be the $j \times j$ leading principal submatrix of the $k_{i} \times k_{i}$ symmetric tridiagonal matrix $X_{i, k_{i}}=a D+$

$$
\left[\begin{array}{ccccc}
\theta_{m_{i, 1}}\left(M_{i, 1}\right) & w_{i, 1} \sqrt{m_{i, 1}} & & & \\
w_{i, 1} \sqrt{m_{i, 1}} & \theta_{m_{i, 2}}\left(M_{i, 2}\right) & \ddots & & \\
& \ddots & \ddots & w_{i, k_{i}-2} \sqrt{m_{i, k_{i}-2}} & \\
& & w_{i, k_{i}-2 \sqrt{m_{i, k_{i}-2}}} & \theta_{m_{i, k_{i}-1}}\left(M_{i, k_{i}-1}\right) & w_{i, k_{i}-1} \sqrt{m_{i, k_{i}-1}} \\
& & & w_{i, k_{i}-1} \sqrt{m_{i, k_{i}-1}} & 0
\end{array}\right],
$$

where $a$ is defined as in (3) and $D=\operatorname{diag}\left(\delta_{i, 1}, \delta_{i, 2}, \ldots, \delta_{i, k_{i}-1}, \delta_{i, k_{i}}\right)$.

Definition 2.2 For $i=1, \ldots, r, j=1, \ldots, k_{i}-1$ and $l=1, \ldots, m_{i, j}-1$, let $X_{i, j, l}=$

$$
\left[\begin{array}{ccccc}
a \delta_{i, 1}+\theta_{m_{i, 1}}\left(M_{i, 1}\right) & w_{i, 1} \sqrt{m_{i, 1}} & & & \\
w_{i, 1} \sqrt{m_{i, 1}} & \ddots & \ddots & & \\
& \ddots & \ddots & w_{i, j}-2 \sqrt{m_{i, j}-2} & \\
& & w_{i, j-2} \sqrt{m_{i, j-2}} & \begin{array}{c}
a \delta_{i, j-1}+\theta_{m_{i, j-1}}\left(M_{i, j-1}\right) \\
w_{i, j-1} \sqrt{m_{i, j-1}}
\end{array} & \begin{array}{c}
w_{i, j-1} \sqrt{m_{i, j-1}} \\
a \delta_{i, j}+\theta_{l}\left(M_{i, j}\right)
\end{array}
\end{array}\right] .
$$

\section{The main results}

Defining $\Omega_{i}=\left\{j: 1 \leq j \leq k_{i}-1, n_{i, j}>n_{i, j+1}\right\}, i=1, \ldots, r$ and applying Theorem 1.2 and Lemma 1.3 to $\mathcal{H}_{i}=\mathcal{B}_{i}\left(F_{i}\right)$ we obtain the following lemma.

Lemma 3.1 For $i=1, \ldots, r$,

$$
\begin{aligned}
\left|\lambda I-M\left(\mathcal{H}_{i}\right)\right| & =D_{i, k_{i}}(\lambda) P_{i}(\lambda) \\
\left|\lambda I-\widehat{M\left(\mathcal{H}_{i}\right)}\right| & =D_{i, k_{i}-1}(\lambda) P_{i}(\lambda)
\end{aligned}
$$

where

$$
P_{i}(\lambda)=\prod_{j \in \Omega_{i}-\Delta_{i}}\left(D_{i, j}(\lambda)\right)^{n_{i, j}-n_{i, j+1}} \prod_{j \in \Delta_{i}} \prod_{l=1}^{m_{i, j}-1}\left(D_{i, j, l}(\lambda)\right)^{n_{i, j+1}}
$$

and for $j=1, \ldots, k_{i}$ and $l=1, \ldots, m_{i, j}-1, D_{i, j}(\lambda)$ and $D_{i, j, l}(\lambda)$ are the characteristic polynomials of the matrices $X_{i, j}$ and $X_{i, j, l}$, respectively.

Before proceeding, it is also worth to recall the following lemma deduced in [10].

Lemma 3.2 [10] For $i=1,2, \ldots, r$, let $B_{i}$ be a matrix of order $k_{i} \times k_{i}$ and $\mu_{i, j}$ 
be arbitrary scalars. Then

$$
\begin{gathered}
\left|\begin{array}{ccccc}
B_{1} & \mu_{1,2} E_{1,2} & \cdots & \mu_{1, r-1} E_{1, r-1} & \mu_{1, r} E_{1, r} \\
\mu_{2,1} E_{1,2}^{T} & B_{2} & \cdots & \cdots & \mu_{2, r} E_{2, r} \\
\mu_{3,1} E_{1,3}^{T} & \mu_{3,2} E_{2,3}^{T} & \ddots & \cdots & \vdots \\
\vdots & \vdots & \vdots & B_{r-1} & \mu_{r-1, r} E_{r-1, r} \\
\mu_{r, 1} E_{1, r}^{T} & \mu_{r, 2} E_{2, r}^{T} & \cdots & \mu_{r, r-1} E_{r-1, r}^{T} & B_{r}
\end{array}\right| \\
=\left|\begin{array}{ccccc}
\left|B_{1}\right| & \mu_{1,2}\left|\widetilde{B_{2}}\right| & \cdots & \mu_{1, r-1}\left|\widetilde{B_{r-1}}\right| & \mu_{1, r}\left|\widetilde{B_{r}}\right| \\
\mu_{2,1}\left|\widetilde{B_{1}}\right| & \left|B_{2}\right| & \cdots & \cdots & \mu_{2, r}\left|\widetilde{B_{2}}\right| \\
\mu_{3,1}\left|\widetilde{B_{1}}\right| & \mu_{3,2}\left|\widetilde{B_{2}}\right| & \ddots & \cdots & \vdots \\
\vdots & \vdots & \vdots & \left|B_{r-1}\right| & \mu_{r-1, r}\left|\widetilde{B_{r-1}}\right| \\
\mu_{r, 1}\left|\widetilde{B_{1}}\right| & \mu_{r, 2}\left|\widetilde{B_{2}}\right| & \cdots & \mu_{r, r-1}\left|\widetilde{B_{r-1}}\right| & \left|B_{r}\right|
\end{array}\right| .
\end{gathered}
$$

The next theorem states the main result of this paper.

Theorem 3.3 Let us consider the graph $\mathcal{G}=\mathcal{R}\{\mathcal{H}\}$. If (4) holds then

$\sigma(M(\mathcal{G}))=\sigma(X) \cup \bigcup_{i=1}^{r}\left(\cup_{j \in \Omega_{i}-\Delta_{i}} \sigma\left(X_{i, j}\right)^{n_{i, j}-n_{i, j+1}} \cup_{j \in \Delta_{i}}\left(\cup_{l=1}^{m_{i, j}-1} \sigma\left(X_{i, j, l}\right)^{n_{i, j+1}}\right)\right)$

where

$$
X=\left[\begin{array}{cccc}
X_{1, k_{1}}+a \varepsilon_{1} E_{1,1} & s \varepsilon_{1,2} E_{1,2} & \cdots & s \varepsilon_{1, r} E_{1, r} \\
s \varepsilon_{1,2} E_{1,2}^{T} & X_{2, k_{2}}+a \varepsilon_{2} E_{2,2} & & \vdots \\
\vdots & \ddots & \ddots & \vdots \\
\vdots & & \ddots & s \varepsilon_{r-1, r} E_{r-1, r} \\
s \varepsilon_{1, r} E_{1, r}^{T} & \cdots & s \varepsilon_{r-1, r} E_{r-1, r}^{T} & X_{r, k_{r}}+a \varepsilon_{r} E_{r, r}
\end{array}\right]
$$

$\sigma\left(X_{i, j}\right)^{n_{i, j}-n_{i, j+1}}$ and $\sigma\left(X_{i, j, l}\right)^{n_{i, j+1}}$ mean that each eigenvalue in $\sigma\left(X_{i, j}\right)$ and in $\sigma\left(X_{i, j, l}\right)$ must be considered with multiplicity $n_{i, j}-n_{i, j+1}$ and $n_{i, j+1}$, respectively. Furthermore, the multiplicities of equal eigenvalues obtained in different matrices (if any), must be added.

Proof. We apply Lemma 3.2 to obtain $|\lambda I-M(\mathcal{G})|=$

$$
\begin{array}{cccc}
\left|\lambda I-M\left(\mathcal{H}_{1}\right)-a \varepsilon_{1} E_{1,1}\right| & -s \varepsilon_{1,2} \mid \lambda I-\widetilde{M\left(\mathcal{H}_{2}\right)} & \cdots & -s \varepsilon_{1, r} \mid \lambda I-\widetilde{M\left(\mathcal{H}_{r}\right)} \\
-s \varepsilon_{1,2}\left|\lambda I-\widetilde{M\left(\mathcal{H}_{1}\right)}\right| & \ddots & \ddots & \vdots \\
\vdots & \ddots & \ddots & \vdots \\
\vdots & \cdots & \ddots & -s \varepsilon_{r-1, r}\left|\lambda I-\widetilde{M\left(\mathcal{H}_{r}\right)}\right| \\
-s \varepsilon_{1, r} \mid \lambda I-\widetilde{M\left(\mathcal{H}_{1}\right)} & \cdots & \left|\lambda I-M\left(\mathcal{H}_{r}\right)-a \varepsilon_{r} E_{r, r}\right|
\end{array}
$$


For $i=1, \ldots, r$, applying linearity on the last column of $\left|\lambda I-M\left(\mathcal{H}_{i}\right)-a \varepsilon_{i} E_{i, i}\right|$, we have

$$
\left.\left|\lambda I-M\left(\mathcal{H}_{i}\right)-a \varepsilon_{i} E_{i, i}\right|=\left|\lambda I-M\left(\mathcal{H}_{i}\right)\right|-a \varepsilon_{i} \mid \lambda I-\widetilde{M\left(\mathcal{H}_{i}\right.}\right) \mid .
$$

Now, using Lemma 3.1, we obtain

$$
\left|\lambda I-M\left(\mathcal{H}_{i}\right)-a \varepsilon_{i} E_{i, i}\right|=\left(D_{i, k_{i}}(\lambda)-a \varepsilon_{i} D_{i, k_{i}-1}(\lambda)\right) P_{i}(\lambda)
$$

Therefore $|\lambda I-M(\mathcal{G})|=$

$$
\left|\begin{array}{cccc}
\left(D_{1, k_{1}}-a \varepsilon_{1} D_{1, k_{1}-1}\right) P_{1} & -s \varepsilon_{1,2} D_{2, k_{2}-1} P_{2} & \cdots & -s \varepsilon_{1, r} D_{r, k_{r}-1} P_{r} \\
-s \varepsilon_{1,2} D_{1, k_{1}-1} P_{1} & \ddots & & \vdots \\
\vdots & \ddots & \ddots & \vdots \\
\vdots & & \ddots & -s \varepsilon_{r-1, r} D_{r, k_{r}-1} P_{r} \\
-s \varepsilon_{1, r} D_{1, k_{1}-1} P_{1} & \cdots & \cdots & \left(D_{r, k_{r}}-a \varepsilon_{r} D_{r, k_{r}-1}\right) P_{r}
\end{array}\right|=
$$

$R(\lambda) \prod_{i=1}^{r} P_{i}(\lambda)$, where $R(\lambda)=$

$$
\begin{array}{cccc}
D_{1, k_{1}}-a \varepsilon_{1} D_{1, k_{1}-1} & -s \varepsilon_{1,2} D_{2, k_{2}-1} & \cdots & -s \varepsilon_{1, r} D_{r, k_{r}-1} \\
-s \varepsilon_{1,2} D_{1, k_{1}-1} & \ddots & \ddots & \vdots \\
\vdots & \ddots & \ddots & \vdots \\
\vdots & & \ddots & -s \varepsilon_{r-1, r} D_{r, k_{r}-1} \\
-s \varepsilon_{1, r} D_{1, k_{1}-1} & \ldots & -s \varepsilon_{r-1, r} D_{r-1, k_{r-1}-1} & D_{r, k_{r}}-a \varepsilon_{r} D_{r, k_{r}-1}
\end{array}
$$

in which, for short, the variable $\lambda$ has been omitted.

Now, applying Lemma 3.2, we have $|\lambda I-X|=$

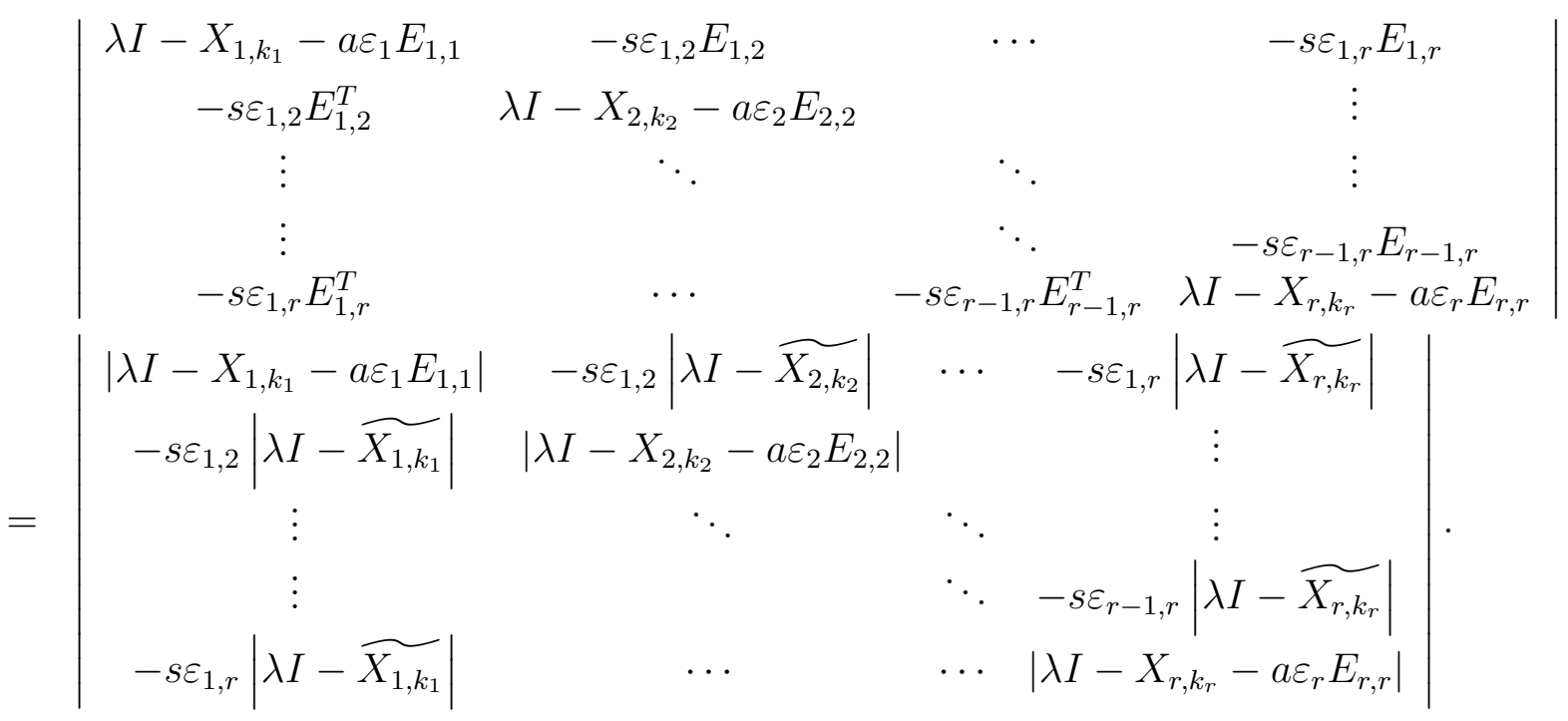


For $i=1, \ldots, r$, expanding along the last column, we get

$$
\left|\lambda I-X_{i, k_{i}}-a \varepsilon_{i} E_{i, i}\right|=\left|\lambda I-X_{i, k_{i}}\right|-a \varepsilon_{i}\left|\lambda I-\widetilde{X_{i, k_{i}}}\right| .
$$

Now, we use the fact that $\left|\lambda I-X_{i, k_{i}}\right|=D_{i, k_{i}}(\lambda)$ and $\left|\lambda I-\widetilde{X_{i, k_{i}}}\right|=D_{i, k_{i}-1}(\lambda)$. Replacing, we obtain $|\lambda I-X|=R(\lambda)$.

\section{The Laplacian, signless Laplacian and adja- cency spectra of $\mathcal{R}\{\mathcal{H}\}$}

Throughout this section, we analyze the particular cases of the Laplacian, signless Laplacian and adjacency spectra of $\mathcal{G}=\mathcal{R}\{\mathcal{H}\}$.

\subsection{On the Laplacian spectra of $\mathcal{G}$}

We already observe that condition (4) holds for the Laplacian matrix. Then we may apply Theorem 3.3 to find the Laplacian spectrum of $\mathcal{G}$.

For $i=1, \ldots, r$ and $j=1, \ldots, k_{i}-1$, let

$$
\mu_{1}\left(M_{i, j}\right) \geq \mu_{2}\left(M_{i, j}\right) \geq \cdots \geq \mu_{m_{i, j}}\left(M_{i, j}\right)=0
$$

where $M_{i, j}=L\left(\mathcal{G}_{i, j}\right)$.

Since, according to $(2)$ and $(3), s=-1$ and $a=1$ whenever $M=L(\mathcal{G})$ and $\mu_{m_{i, j}}\left(M_{i, j}\right)=0$ for $i=1, \ldots, r$ and $j=1, \ldots, k_{i}-1$, the Laplacian spectrum of $\mathcal{G}$ follows easily from Theorem 3.3.

Theorem 4.1 The Laplacian spectrum of $\mathcal{G}=\mathcal{R}\{\mathcal{H}\}$ is

$\sigma(L(\mathcal{G}))=\sigma(U) \cup \bigcup_{i=1}^{r}\left(\cup_{j \in \Omega_{i}-\Delta_{i}} \sigma\left(U_{i, j}\right)^{n_{i, j}-n_{i, j+1}} \cup_{j \in \Delta_{i}}\left(\cup_{l=1}^{m_{i, j}-1} \sigma\left(U_{i, j, l}\right)^{n_{i, j+1}}\right)\right)$

where,

1. for $i=1, \ldots, r$ and $j \in \Omega_{i}-\Delta_{i}, U_{i, j}$ is the $j \times j$ leading principal submatrix of the $k_{i} \times k_{i}$ matrix $U_{i, k_{i}}=X_{i, k_{i}}$ (in this particular case, the diagonal entries in $U_{i, k_{i}}$ are $\left.\delta_{i, 1}, \ldots, \delta_{i, k_{i}}\right)$,

2. for $i=1, \ldots, r, j \in \Delta_{i}$ and $l=1, \ldots, m_{i, j}-1, U_{i, j, l}=X_{i, j, l}$ (in this particular case, the diagonal entries in $U_{i, j, l}$ are $\delta_{i, 1}, \ldots, \delta_{i, j-1}, \delta_{i, j}+\mu_{l}\left(L\left(\mathcal{G}_{i, j}\right)\right)$ ),

3. and $U=X$, with $X_{i, k_{i}}=U_{i, k_{i}}, a=1$ and $s=-1$.

The multiplicities of the eigenvalues of $L(\mathcal{G})$ are considered as in Theorem 3.3. 


\subsection{On the signless Laplacian and adjacency spectra of $\mathcal{G}$}

We denote the signless Laplacian eigenvalues and adjacency eigenvalues of a graph $\mathcal{S}$ of order $m$ as follows

$$
q_{1}(\mathcal{S}) \leq q_{2}(\mathcal{S}) \leq q_{3}(\mathcal{S}) \leq \cdots \leq q_{m-1}(\mathcal{S}) \leq q_{m}(\mathcal{S})
$$

and

$$
\lambda_{1}(\mathcal{S}) \leq \lambda_{2}(\mathcal{S}) \leq \cdots \leq \lambda_{m-1}(\mathcal{S}) \leq \lambda_{m}(\mathcal{S})
$$

respectively. In particular, if $\mathcal{S}$ is a regular graph of degree $t$ and order $m$ in which the edges have a weight equal to $u$ then $Q(\mathcal{S}) \mathbf{e}_{m}=2 t u \mathbf{e}_{m}, A(\mathcal{S}) \mathbf{e}_{m}=t u \mathbf{e}_{m}$ and thus $q_{m}(\mathcal{S})=2 t u$ and $\lambda_{m}(\mathcal{S})=t u$.

If each $\mathcal{G}_{i, j}$ is a regular graph of degree $r_{i, j}$ then (4) holds and we may apply Theorem 3.3 to obtain the eigenvalues of $Q(\mathcal{G})$ and $A(\mathcal{G})$.

Since, according to (2) and (3), $s=1$ and $a=1$ whenever $M=Q(G)$ and $q_{m_{i, j}}\left(M_{i, j}\right)=2 m_{i, j} u_{i, j}$ for $i=1, \ldots, r$ and $j=1, \ldots, k_{i}-1$, the signless Laplacian spectrum of $\mathcal{G}$ follows easily from Theorem 3.3.

Theorem 4.2 If for $i=1, \ldots, r$ and for each $j \in \Delta_{i}$ the graph $\mathcal{G}_{i, j}$ is a regular graph of degree $r_{i, j}$ and $r_{i, j}=0$ whenever $j \notin \Delta_{i}$, then the signless Laplacian spectrum of $\mathcal{G}=\mathcal{R}\left\{\mathcal{H}_{i}: 1 \leq i \leq r\right\}$ is

$\sigma(Q(\mathcal{G}))=\sigma(V) \cup \bigcup_{i=1}^{r}\left(\cup_{j \in \Omega_{i}-\Delta_{i}} \sigma\left(V_{i, j}\right)^{n_{i, j}-n_{i, j+1}} \cup_{j \in \Delta_{i}}\left(\cup_{l=1}^{m_{i, j}-1} \sigma\left(V_{i, j, l}\right)^{n_{i, j+1}}\right)\right)$

where,

1. for $i=1, \ldots, r$ and $j \in \Omega_{i}-\Delta_{i}, V_{i, j}$ is the $j \times j$ leading principal submatrix of the $k_{i} \times k_{i}$ matrix $V_{i, k_{i}}=X_{i, k_{i}}$ (in this particular case the diagonal entries in $V_{k_{i}}$ are $\left.\delta_{i, 1}+2 r_{i, 1} u_{i, 1}, \delta_{i, 2}+2 r_{i, 2} u_{i, 2}, \ldots, \delta_{i, k_{i}-1}+2 r_{i, k_{i}-1} u_{i, k_{i}-1}, \delta_{i, k_{i}}\right)$,

2. for $i=1, \ldots, r, j \in \Delta_{i}$ and $l=1, \ldots, m_{i, j}-1, V_{i, j, l}=X_{i, j, l}$ (in this particular case the diagonal entries in $V_{i, j, l}$ are $\delta_{i, 1}+2 r_{i, 1} u_{i, 1}, \delta_{i, 2}+2 r_{i, 2} u_{i, 2}, \ldots, \delta_{i, j-1}+$ $\left.2 r_{i, j-1} u_{i, j-1}, \delta_{i, j}+q_{l}\left(\mathcal{G}_{i, j}\right)\right)$,

3. and $V=X$, with $X_{i, k_{i}}=V_{i, k_{i}}, a=1$ and $s=1$.

The multiplicities of the eigenvalues of $Q(\mathcal{G})$ are considered as in Theorem 3.3.

Since, according to (2) and (3), $s=1$ and $a=0$ whenever $M=A(\mathcal{G})$ and $\lambda_{m_{i, j}}\left(M_{i, j}\right)=m_{i, j} u_{i, j}$ for $i=1, \ldots, r$ and $j=1, \ldots, k_{i}-1$, the application of Theorem 3.3 yields to 
Theorem 4.3 If for $i=1, \ldots, r$ and for each $j \in \Delta_{i}$ the graph $\mathcal{G}_{i, j}$ is a regular graph of degree $r_{i, j}$ and $r_{i, j}=0$ whenever $j \notin \Delta_{i}$, then the adjacency spectrum of $\mathcal{G}=\mathcal{R}\{\mathcal{H}\}$ is $\sigma(A(\mathcal{G}))=\sigma(W) \cup \bigcup_{i=1}^{r}\left(\cup_{j \in \Omega_{i}-\Delta_{i}} \sigma\left(W_{i, j}\right)^{n_{i, j}-n_{i, j+1}} \cup_{j \in \Delta_{i}} \cup_{l=1}^{m_{i, j}-1} \sigma\left(W_{i, j, l}\right)^{n_{i, j+1}}\right)$ where,

1. for $i=1, \ldots, r$ and $j \in \Omega_{i}-\Delta_{i}, W_{i, j}$ is the $j \times j$ leading principal submatrix of the $k_{i} \times k_{i}$ matrix $W_{i, k_{i}}=X_{i, k_{i}}$ (in this particular case the diagonal entries in $W_{i, k_{i}}$ are $\left.r_{i, 1} u_{i, 1}, r_{i, 2} u_{i, 2}, \ldots, r_{i, k_{i}-1} u_{i, k_{i}-1}, 0\right)$,

2. for $i=1, \ldots, r, j \in \Delta_{i}$ and $l=1, \ldots, m_{i, j}-1, W_{i, j, l}=X_{i, j, l}$ (in this particular case the diagonal entries in $W_{i, j, l}$ are $r_{i, 1} u_{i, 1}, r_{i, 2} u_{i, 2}, \ldots, r_{i, j-1} u_{i, j-1}$, $\left.\lambda_{l}\left(\mathcal{G}_{i, j}\right)\right)$,

3. and $W=X$, with $X_{i, k_{i}}=W_{i, k_{i}}, a=0$ and $s=1$.

The multiplicities of the eigenvalues of $A(\mathcal{G})$ are considered as in Theorem 3.3.

From Theorem 4.1, Theorem 4.2 and Theorem 4.3, the Laplacian eigenvalues, signless Laplacian eigenvalues and adjacency eigenvalues of $\mathcal{R}(\mathcal{H})$, respectively, are the eigenvalues of symmetric tridiagonal matrices of order $j$, $1 \leq j \leq \max \left\{k_{1}, \ldots, k_{r}\right\}$. Thus we may use the stable and low computational cost methods available to compute the eigenvalues of such matrices.

Acknowledgment. The research of E. Andrade and D. M. Cardoso was supported by Project Fondecyt Regular 1130135, Chile and by the Portuguese Foundation for Science and Technology (FCT-Fundação para a Ciência e a Tecnologia), through CIDMA - Center for Research and Development in Mathematics and Applications, within project UID/MAT/04106/2013. These authors also thank the hospitality of Departamento de Matemáticas, Universidad Católica del Norte, Antofagasta. L. Medina thanks the hospitality of the Departamento de Matemática, Universidade de Aveiro, Aveiro, Portugal, in which part of this research was conducted. The research of O. Rojo was supported by Project Fondecyt Regular 1130135, Chile. This author thanks the hospitality of the Departamento de Matemática, Universidade de Aveiro, Aveiro, Portugal, and the hospitality of the Center For Mathematical Modeling, Universidad de Chile, Chile, in which part of this research was conducted.

\section{References}

[1] S. Beigi, I. Chuang, M. Grassl, P. Shor, and B. Zeng, Graph Concatenation for Quantum Codes, J. Math. Phys. 52, 022201 (2011). 
[2] D. M. Cardoso, E. A. Martins, M. Robbiano, O. Rojo, Copies of a rooted weighted graph attached to an arbitrary weighted graph and applications, Electron. J. Linear Algebra, 26:706-717, 2013.

[3] D. M. Cardoso, E. A. Martins, M. Robbiano, V. Trevisan, Computing the Laplacian spectra of some graphs, Discrete Appl. Math., 160(18):2645-2654, 2012.

[4] B. Courcelle and J. Engelfriet, Graph Structure and Monadic Second-Order Logic, a Language Theoretic Approach, Encyclopedia of Mathematics and its Applications, Cambridge University Press, 2012.

[5] D. Cvetković, P. Rowlinson and S. Simić, An Introduction to the Theory of Graph Spectra, Cambridge University Press, Cambridge, 2010.

[6] B. N. Datta, Numerical Linear Algebra and Applications, Brooks/Cole Publishing Company, 1995.

[7] R. Fernandes, H. Gomes, E. A. Martins, On the spectra of some graphs like weighted rooted trees, Linear Algebra Appl. 428 (2008) 2654-2674.

[8] X. Li, Y. Shi, I. Gutman, Graph Energy, Springer, 2012.

[9] O. Rojo, R. Soto, The spectra of the adjacency matrix and Laplacian matrix for some balanced trees, Linear Algebra Appl. 403 (2005) 97-117.

[10] O. Rojo, L. Medina, Spectra of weighted compounds graphs of generalized Bethe trees, Electron. J. Linear Algebra, 18:30-57, 2009.

[11] O. Rojo, L. Medina, Spectral characterization of some weighted rooted graphs with cliques, Linear Algebra Appl., 433:1388-1409, 2010.

[12] O. Rojo, M. Robbiano, D. M. Cardoso, E. A. Martins, Spectra of weighted rooted graphs having prescribed subgraphs at some levels, Electron. J. Linear Algebra, 22:653-671, 2011.

[13] F. Zhang, Matrix Theory, Springer, New York, 1999. 